\title{
On the Futile Existence of DHA, None of EPA and the Predominant Role of the Triacylglycerols (TGs) in the Post Mortem Human Brain: An LCMS Study with Evolutionary Implications
}

\author{
Vincent van Ginneken ${ }^{1,2^{*}}$, Aline van Meerveld ${ }^{1}$, Elwin Verheij ${ }^{2}$ and Jan van der Greef ${ }^{2,3}$ \\ ${ }^{1}$ Blue Green Technologies, Runderweg 6, 8219 PK Lelystad, The Netherlands \\ ${ }^{2}$ Netherlands Organisation for Applied Scientific Research (TNO), Utrechtseweg, AJ Zeist, The Netherlands
}

${ }^{3}$ Sino-Dutch Center for Preventive and Personalized Medicine; Leiden University, RA Leiden, The Netherlands

\begin{abstract}
What is known about the brain is a scanty amount of information despite its importance in human evolution. Here we perform based on a holistic Systems Biology lipidomics approach an attempt describing the brain biochemical lipid composition based on the homogenate of a part (gyrus) of the neocortex of post mortem material of the "Dutch Brain Bank" with LCMS techniques. From evolutionary perception the occurrence of the "fish oils" in blood and/ or brain is interesting and their ability to pass the Blood-Brain Barrier (BBB). Both omega-3 "fish oil" Fatty Acids (FA) Eicosapentaenoic Acid (EPA) and Docosahexaenoic Acid (DHA). EPA and DHA are found in rather high concentrations respectively $0.277 \%$ and $0.258 \%$ respectively in blood plasma but not in the brain. EPA is not found in grey or white neocortex matter so probably it cannot pass the BBB while this is the case for DHA giving values of respectively $\approx 0.00015 \%$ while Triacylglycerols (TGs) are the major constituent of the human brain with a mean value of the neocortex of $\approx 72 \%$. We finally conclude the TGs have played a major role in the process of human brain growth and encephalization during course of evolution.
\end{abstract}

Keywords: Human brain; Post mortem; LCMS; Lipids; Blood-Brain Barrier (BBB); Fatty acids; Triacylglycerols (TGs); Human evolution; Diet; Savannah phase; Out of Africa; Encephalization

\section{Introduction}

The neocortex is the part of the mammalian brain involved in higherorder brain functions and it is in general acknowledged this is the brain part which makes us human [1]. We can learn about the evolution of the human brain through archaeological findings by the skull endocasts of fossil archaic primates and early hominids [2] but we can also study biochemical composition with modern LCMS techniques [3] in order to attempt unravel evolution. Previously, it was believed the brain made all the fat it needed itself but a landmark study [4] discovered that Mfsd2a protein is the transporter for LPCs and carrier of the "fish" omega-3 DHA across the Blood-Brain Barrier (BBB). However DHA is in such a scanty amount $(<0.05 \%)$ in the neocortex it can never explain the tremendous growth of Homo sapiens brain. A mouse model with overgrown brain demonstrated this could be attributed to specific Triacylglycerols (TGs) from bovine lard [5] directing to a meat source from savannah. Here we present the lipid composition of modern human brain and its possible places of transport of "Very Long Chain Fatty Acids (VLCFA)" across the "leaky" BBB which can fuel the brain. These new findings give a new perception on evolution with emphasis on the TGs for skull expansion and not solely DHA. We hypothesized in a previous manuscript early hominids lived at the savannah following the herds of early African buffaloes (direct hunter-prey correlation) and that accumulation of specific TGs from bovine lard could have contributed to encephalization in human evolution [6].

Briefly, the human brain is made up of about $75 \%$ water while it is the fattest organ in our body, a $60 \%$ of the brain structural material (dry weight) is lipid [7]. In addition, it contains the highest concentration of lipids, immediately after adipose tissue [8]. Brain lipids are not similar to White Adipose Tissue (WAT) because a large part of the "fat" in the brain is in the form of myelin membrane, which insulates the axons of neurons. The myelin membrane is an extended and highly specialized plasma membrane synthesized by myelinating glial cells:
Oligo-dendrocytes in the Central Nervous System (CNS) and Schwann cells in the Peripheral Nervous System (PNS) [9]. These specialized cells are almost entirely composed of extra cellular membrane, and thus have a high lipid content which gives the brain as a whole a high lipid concentration because of these structural lipid compounds which cannot be catabolized.

The myelin fat content has already been determined for the adult rat in 1973 [9], but for human brain tissue no studies have been performed on this topic. In addition, biochemical data on the lipid composition of the human brain are rare or old-fashioned [10].

What is known about the brain is a scanty amount of information despite its importance in human evolution. From fossil record in early Pleistocene cranial capacity increased over a period of 700,000 years from $850 \mathrm{~cm}^{3}$ towards $\approx 1500 \mathrm{~cm}^{3}$ (Figure 1) [11] which is equivalent to every generation having an additional 125,000 neurons more than their parents, but what were the building blocks for these neurons? It would be a daunting task to describe the human brain based on biochemical composition of each cell type. Here we perform based on a holistic Systems Biology lipidomics approach an attempt describing the brain biochemical lipid composition based on the homogenate of a part (gyrus) of the neocortex of post mortem material of the "Dutch Brain Bank" [12] with LCMS techniques [5,12]. From evolutionary perception

*Corresponding author: Vincent van Ginneken, Blue Green Technologies, Runderweg 6, 8219 PK Lelystad, The Netherlands, Tel: 310638071180; E-mail: vvanginneken@hotmail.com

Received June 03, 2017; Accepted June 17, 2017; Published June 23, 2017

Citation: Ginneken VV, Meerveld AV, Verheij E, Greef JVD (2017) On the Futile Existence of DHA, None of EPA and the Predominant Role of the Triacylglycerols (TGs) in the Post Mortem Human Brain: An LCMS Study with Evolutionary Implications. J Bioanal Biomed 9: 152-158. doi:10.4172/1948-593X.1000170

Copyright: (c) 2017 Ginneken VV, et al. This is an open-access article distributed under the terms of the Creative Commons Attribution License, which permits unrestricted use, distribution, and reproduction in any medium, provided the original author and source are credited. 
Citation: Ginneken VV, Meerveld AV, Verheij E, Greef JVD (2017) On the Futile Existence of DHA, None of EPA and the Predominant Role of the Triacylglycerols (TGs) in the Post Mortem Human Brain: An LCMS Study with Evolutionary Implications. J Bioanal Biomed 9: 152-158. doi:10.4172/1948-593X.1000170

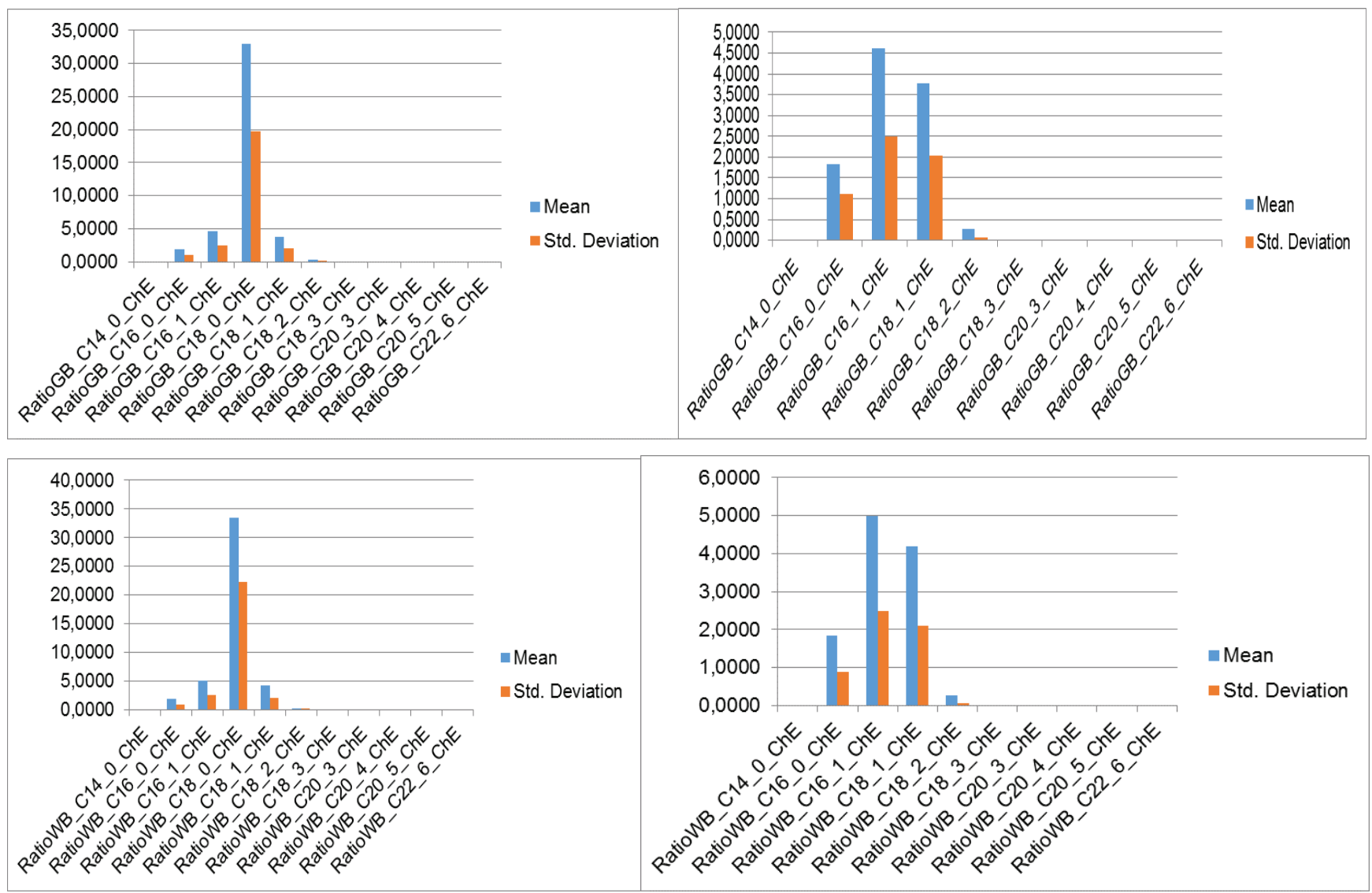

Figure 1: (a \& b) Depicted ration [brain-ChEs] / [blood-ChEs] for grey neocortical matter. Right panel without the dominant C:18-0 peak (stearic acid). Mean (blue) and red (std) of 8 Control Human subjects. (c and d) Depicted ration [brain-ChEs] / [blood-ChEs] for white neocortical matter. Right panel without the dominant C:18-0 peak. Mean (blue) and red (std) of 8 Control Human subjects.

the occurrence of the "fish oils" in blood and/or brain is interesting and their ability to pass the BBB. Based on our observations we open the debate which lipid class-the PUFAs vs. the TGs-have played a major unambiguously role in the process of human brain growth and encephalization. Our initial hypothesis is that the TGs have played a major role in human brain skull expansion or encephalization and that the "fish oil" DHA is a tiny fraction of the FA in the brain and is of minor importance of human encephalization.

\section{Material and Methods}

We quantified and qualified the rearrangement and repartitioning of these fat stores in the brain of male post mortem subjects (Control $\mathrm{n}=8 ; 73.5 \pm 10.5$ years; Type- 2 diabetes $\mathrm{n}=8 ; 77.3 \pm 7.2$ years) which were obtained from the 'Netherlands Brain Bank'. We quantified 109 lipid compounds based on mass and polarity using LCMS methods as described earlier [5,12]. Briefly, we followed next protocol.

Brain-tissue: Human Brain tissue was obtained from the "Netherlands Brain Bank". Causes of death causes are presented in Table 1 [12]. Time of post mortem dissection varied between 4.4 and $24.5 \mathrm{~h}$ (Table 1) [12]. The region of post mortem collection of the tissue material was mainly the cortex (Table 1) [12]. Visually, a clear separation could be made between grey and white matter in the cortex. A tissue homogenate of the brain tissue (grey or white; $10 \%$ wet weight/volume) in Phosphate-Buffered Saline (PBS) was made with the Mini-beat Beater.

Blood plasma: Blood was centrifuged at $8000 \times g$ for $5 \mathrm{~min}$. The plasma was aliquoted and stored at $-80^{\circ} \mathrm{C}$ at the "Netherlands Brain Bank" pending analysis

LC-MS: Lipids and free fatty acids (FFA) were analyzed with electrospray LC-MS as earlier performed. For blood plasma $10 \mu \mathrm{l}$ samples were extracted with $300 \mu \mathrm{l}$ of isopropanol (IPA) containing several internal standards (IS: C17:0 lysophosphatidylcholine, di-C12:0 phosphatidylcholine, tri-C17:0 glycerol ester, C17:0 cholesterol ester and heptadecanoic acid (C17:0)). Fifty $\mu \mathrm{L}$ of the brain homogenate was mixed with $1000 \mu \mathrm{L}$ IPA containing 4 internal standards. After mixing and centrifugation the supernatant was transferred to an autosampler vial. Lipids were separated on a $150 \times 3.2 \mathrm{~mm}$ i.d. C4 Prosphere column (Alltech, USA) using a methanol gradient in $5 \mathrm{mM}$ ammonium acetate and $0.1 \%$ formic acid (mobile phase A: $5 \%$ methanol, mobile phase B: $90 \%$ methanol). The flowrate was $0.4 \mathrm{ml} / \mathrm{min}$ and the gradient was as follows: $0-2$ min- $20 \%$ B, $2-3$ min- $20 \%$ to $80 \%$ B, 3- 15 min- $80 \%$ to $100 \%$ B, 15-25 min-hold $100 \%$ B, 25-32 min-condition at 20\% B.

The instrument used was a Thermo LTQ equipped with a Thermo Surveyor HPLC pump. Data were acquired by scanning the instrument from m/z 300 to 1200 at a scan rate of approximately 2 scans/s in positive ion ESI mode. 
Citation: Ginneken VV, Meerveld AV, Verheij E, Greef JVD (2017) On the Futile Existence of DHA, None of EPA and the Predominant Role of the Triacylglycerols (TGs) in the Post Mortem Human Brain: An LCMS Study with Evolutionary Implications. J Bioanal Biomed 9: 152-158. doi:10.4172/1948-593X.1000170

The FFA LC-MS platform employs the same sample and similar HPLC conditions as the lipid method. The ammonium acetate concentration is $2 \mathrm{mM}$ instead of $5 \mathrm{mM}$ and no formic acid was added. The gradient: $0-2$ min- $30 \% \mathrm{~B}, 2-3 \mathrm{~min}-30 \%$ to $70 \% \mathrm{~B}, 3-10 \mathrm{~min}-70 \%$ to $100 \% \mathrm{~B}, 10-15$ min-hold $100 \% \mathrm{~B}, 15-20$ min-condition at $30 \% \mathrm{~B}$. Detection of FFA is performed in negative ion ESI mode. Combined the two methods provide (semi)quantitative data for approximately 200 different identified lipids and FFA.

Each extract was injected three times $(10 \mu \mathrm{l})$, once for the LC-MS FFA platform and two times for the LC-MS lipid platform. Furthermore, a Quality Control (QC) sample was prepared by pooling the samples. The pool was divided into $10 \mu \mathrm{l}$ aliquots that were extracted the same as the study samples. The QC samples were placed at regular intervals in the analysis sequence (one QC after every 10 samples). The QC samples served two purposes. The first is a regular quality control sample to monitor the LC-MS response in time. After the response has been characterized, the QC samples were used as standards of unknown composition to calibrate the data.

In plasma samples, the 6 dominant lipid classes observed with these two methods are the lyso-phosphatidylcholines (IS used: C17:0 lyso-phosphatidylcholine), phosphatidylcholines (IS used: di-C12:0 phosphatidylcholine), sphingomyelines (IS used: di-C12:0 phosphatidylcholine), cholesterylesters (IS used: C17:0 cholesterylester), triacylglycerols (IS used: tri-C17:0 glycerol ester), and free fatty acids (IS used: C17:0 FFA) (Figure 1a and 1b; Annex 1). In addition to these lipids, the extracts also contain minor lipids, but these were either not detected (concentration too low relative to very abundant lipids like phosphatidylcholines and diacylglycerols, DG) or they were not included in data processing with exception of the DG. The LC-MS lipid and LC-MS FFA data were processed using the LC-Quan software (Thermo).

Analysis of plasmalogens: Analysis of Cholesterylesters (ChE), Lysophosphatidyl-cholines (LPC), Phosphatidylcholine (PC), Sphingomyelin (SPM) and Triacylglycerols (TGs) were based on molecular mass and retention time using internal standards. Because no standards for the plasmalogens exist, mass lists determined by using Atmospheric Pressure Chemical Ionization (APCI) and Electrospray Ionization (ESI), Mass Spectrometry (MS) techniques were used. These mass lists were published on internet www.byrdwell.com/plasmogens and www.byrdwell.com/-PhosphatidylEthanolamine.
Calculations "Hot-spot" passage Blood-Brain Barrier: Next we calculated at the molecular level for every individual lipid in blood plasma vs. brain across the Blood-Brain Barrier for Cholesterylesters and Triacylglycerols ratios following:

- Ration [brain-ChEs]/[blood-ChEs] for grey neocortical matter vs. white neocortical matter.

- Ration [brain-TGs]/[blood-TGs] for white neocortical matter vs. grey neocortical matter.

Statistics: For all measured parameters the mean value of the control post-mortem human group was compared to the mean value of the post-mortem T2DM human group. Statistics were performed via SPSS using one-way ANOVA for differences between control and T2DM groups. $\mathrm{P} \leq 0.05$ was considered as statistically significant. Normality of the data and homogeneity of variances were checked by KolmogorovSmirnov and $\mathrm{F}_{\max }$ tests, respectively $[5,12]$.

\section{Results}

From Table 1 no significant differences in the major lipid classes (MG, LPC, SPM, PC, DG, ChE, TG) in blood plasma or the ratios AA/ DHA and AA/EPA ratios were observed between the experimental groups. Because we measured for every patient directly linked the biochemical brain homogenate (grey and white) composition together with blood plasma this enables us to calculate the gradient for every lipid molecule across the BBB for Cholesterylesters (ChEs Figure 1a1d) and Triacylglycerols (TGs Figure 2a-2d).

For blood plasma (Table 1) our major findings were that PCs are the major constituent (44.36\%) followed by the by diet-influenced TGs $(27.9 \%)$. The PUFAs ( $\approx$ Cholesterylesters) take an important place (13.61\%) as well as their carriers the LPC lipid compound $(5.64 \%)$ [13-16]. Interestingly, both omega-3 "fish oil" FA EPA and DHA are found in rather high concentrations respectively $0.277 \%$ and $0.258 \%$ respectively in blood plasma but not in the brain. EPA is not found in grey or white neocortex matter so probably it cannot pass the bloodbrain barrier (BBB) while this is the case for DHA giving values of respectively $0.00014 \%$ vs. $0.000162 \%$ for white and grey matter $(\mathrm{P} \leq$ 0.213; Table 2).

Another important observation is that the TGs are the major

\begin{tabular}{|c|c|c|c|c|c|}
\hline Compound & Control human blood plasma Mean \pm SD $(n=8)$ & $\%$ & Type-2 diabetes human blood plasma Mean \pm SD $(n=8)$ & $\%$ & T-test P-value \\
\hline$\sum-M G$ & $0.172 \pm 0.070$ & $0.42 \%$ & $0.339 \pm 0.218$ & $0.68 \%$ & $P \leq 0.058$ \\
\hline$\sum-\mathrm{LPC}$ & $2.320 \pm 1.778$ & $5.64 \%$ & $1.566 \pm 0.560$ & $3.15 \%$ & $P \leq 0.272$ \\
\hline$\sum$-SPM & $3.051 \pm 1.485$ & $7.42 \%$ & $3.862 \pm 1.280$ & $7.78 \%$ & $P \leq 0.261$ \\
\hline$\sum-\mathrm{PC}$ & $18.252 \pm 6.828$ & $44.36 \%$ & $19.688 \pm 5.617$ & $39.64 \%$ & $P \leq 0.653$ \\
\hline$\sum$-DG & $0.271 \pm 0.215$ & $0.66 \%$ & $0.533 \pm 0.541$ & $1.07 \%$ & $P \leq 0.224$ \\
\hline$\sum-\mathrm{ChE}$ & $5.598 \pm 2.695$ & $13.61 \%$ & $6.293 \pm 1.704$ & $12.67 \%$ & $P \leq 0.548$ \\
\hline$\sum-\mathrm{TG}$ & $11.478 \pm 7.070$ & $27.90 \%$ & $17.390 \pm 14.070$ & $35.01 \%$ & $P \leq 0.306$ \\
\hline$\sum$-Total blood & 41.142 & $100.0 \%$ & 49.671 & $100.0 \%$ & \\
\hline DGLA (C20:3n6) & $0.070 \pm 0.054$ & $0.170 \%$ & $0.078 \pm 0.043$ & $0.157 \%$ & $P \leq 0.754$ \\
\hline AA (C:20-4-ChE) & $0.983 \pm 0.620$ & $2.389 \%$ & $1.354 \pm 0.671$ & $2.726 \%$ & $P \leq 0.270$ \\
\hline EPA (C:20-5-ChE) & $0.114 \pm 0.131$ & $0.277 \%$ & $0.186 \pm 0.111$ & $0.374 \%$ & $P \leq 0.251$ \\
\hline DHA (C:22-6-ChE) & $0.106 \pm 0.080$ & $0.258 \%$ & $0.170 \pm 0.083$ & $0.342 \%$ & $P \leq 0.134$ \\
\hline AA/DHA & $11.52 \pm 5.085$ & - & $8.17 \pm 2.166$ & - & $P \leq 0.108$ \\
\hline AA/EPA & $14.01 \pm 7.903$ & - & $9.07 \pm 5.184$ & - & $P \leq 0.162$ \\
\hline
\end{tabular}

Table 1: Summarized relative concentrations determined with LC-MS techniques of the different lipid compounds in human blood plasma of two patients groups: one Control-group ( $\mathrm{N}=8$ ) and one Diabetes-2 group ( $\mathrm{N}=8)$. DGLA: Dihomo-y-linolenic Acid; AA: Arachidonic Acid; EPA: Eicosapentaenoic Acid; DHA: Docosahexaenoic Acid. 
Citation: Ginneken VV, Meerveld AV, Verheij E, Greef JVD (2017) On the Futile Existence of DHA, None of EPA and the Predominant Role of the Triacylglycerols (TGs) in the Post Mortem Human Brain: An LCMS Study with Evolutionary Implications. J Bioanal Biomed 9: 152-158. doi:10.4172/1948-593X.1000170

constituent of the human brain for white vs. grey respectively $68.32 \mathrm{vs.}$ $75.96 \%$ respectively $(\mathrm{P} \leq 0.968)$ with a mean value of the neocortex of $\approx 72 \%$. So the [brain-TGs]/[blood-TGs] ratio $=72 \% / 28 \%=2.57$.

This is in general so important PUFAs ( $\approx \mathrm{ChE})$ exemplify solely a minor lipid fraction in the human brain giving values of respectively $0.05 \%$ vs. $0.05 \%$ for white and grey matter $(\mathrm{P} \leq 0.05$; Table 2$)$, giving a mean value of the neocortex of $\approx 0.05 \%$. So the [brain-ChE]/[bloodChEs] ratio $=0.05 \% / 13.61 \%=0.0037$.

Brain phospholipids are distinctive from in that they tend to contain a higher proportion of phosphatidylcholine $(\approx 18.8 \%)$ and much lower values for the phosphatidylethanolamine $(\approx 1.3 \%)$ especially in plasmalogen form ( $\sum$-PC-plasmalogens and $\sum$-PE-plasmalogens between $\approx 1-2 \%$; Table 2).

Next we calculated at the molecular level for every individual lipid in blood plasma vs. brain across the BBB similar ratios which are depicted in Figure 1 ( $\mathrm{a}, \mathrm{b}$ vs. c, d). From these observations we can conclude from the in Figure 1 depicted ration [brain-ChEs]/[bloodChEs] for grey neocortical matter vs. white neocortical matter that the $\mathrm{C} 18: 0 \mathrm{ChE}$ ration (stearic acid) is the most abundant so the $\mathrm{BBB}$ is in theory the most "leaky" for this ChE (relative range grey $\approx 33.0$ vs. white $\approx 33.5$ ) (left panels). If we eliminate this peak the most abundant ChEs for grey (Figure 1b) and white (Figure 1d) are C16:0; C16:1; C18:1 ChEs (range $\approx 1.8-5.0$ ).

If we consider the TGs fraction (Figure $2 \mathrm{a}$ and $2 \mathrm{~b}$ white $v s$. Figure $3 \mathrm{a}$ and $3 \mathrm{~b}$ grey) with depicted ration [brain-TGs]/[blood-TGs] for white neocortical matter vs. grey neocortical matter we can conclude that C:54-1 TG is the most abundant peak $\approx 83000.0$ (white) $\approx 78000.0$ (grey) so the BBB is the most "leaky" for this lipid molecule. If we eliminate this optional important evolutionary peak we see depicted in Figure 2 (b vs. d) that the BBB is "leaky" for a whole range of TGs, to mention in the range from $C: 46-C: 60$ unsaturated and saturated TGs which may have contribute for further brain growth.

\section{Discussion and Conclusion}

Recent investigations are indicative that brain health could be associated with many different physiological and pathophysiological

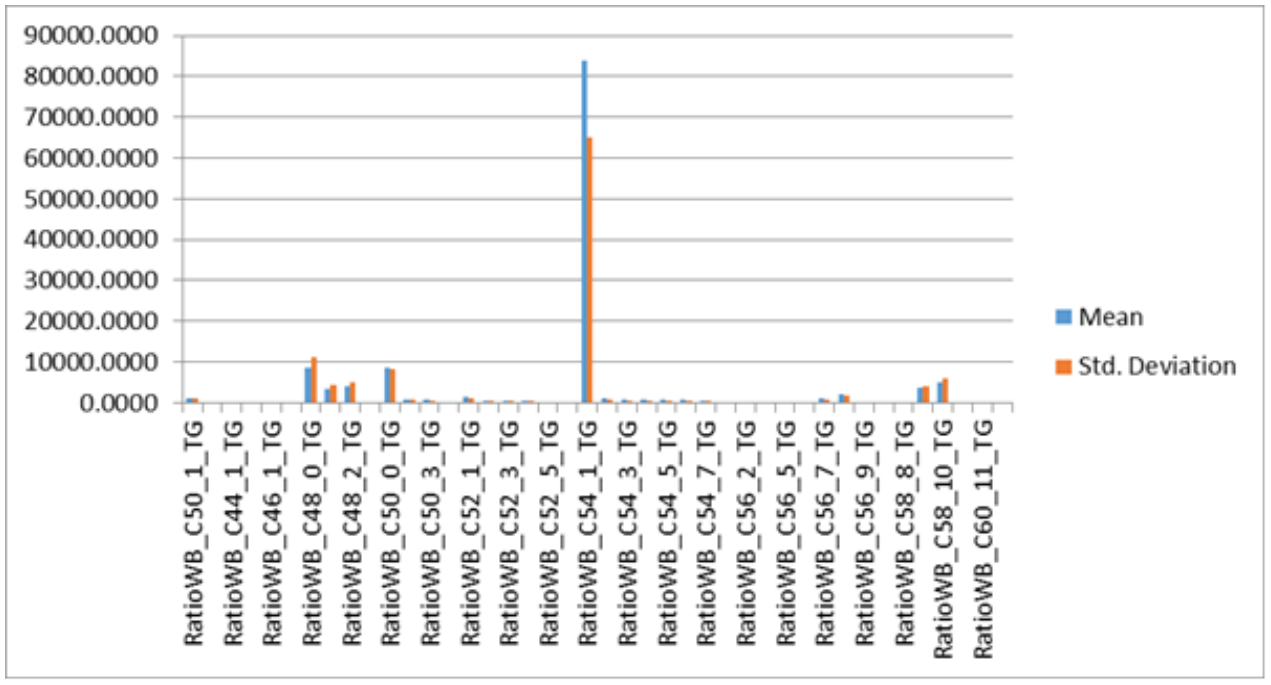

(a)

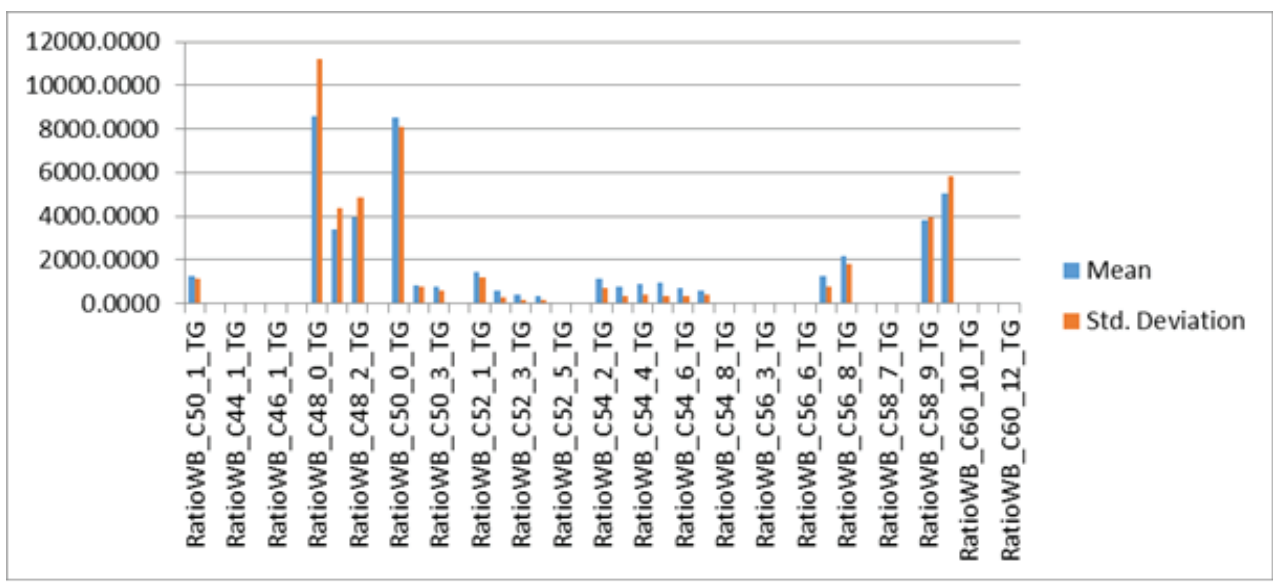

(b)

Figure 2: With depicted ration [brain-TGs]/[blood-TGs] for white neocortical matter. When from Figure $2 a$ (top) the dominant TG-C:54-1 peak $\approx 83000.0$ is eliminated we get in Figure $2 \mathrm{~b}$ (bottom) variety of TGs fatty acids fatty acids of varying degrees of unsaturation and chain length which possibly fuel the brain. Mean (blue) and red (std) of 8 control human subjects 
Citation: Ginneken VV, Meerveld AV, Verheij E, Greef JVD (2017) On the Futile Existence of DHA, None of EPA and the Predominant Role of the Triacylglycerols (TGs) in the Post Mortem Human Brain: An LCMS Study with Evolutionary Implications. J Bioanal Biomed 9: 152-158. doi:10.4172/1948-593X.1000170
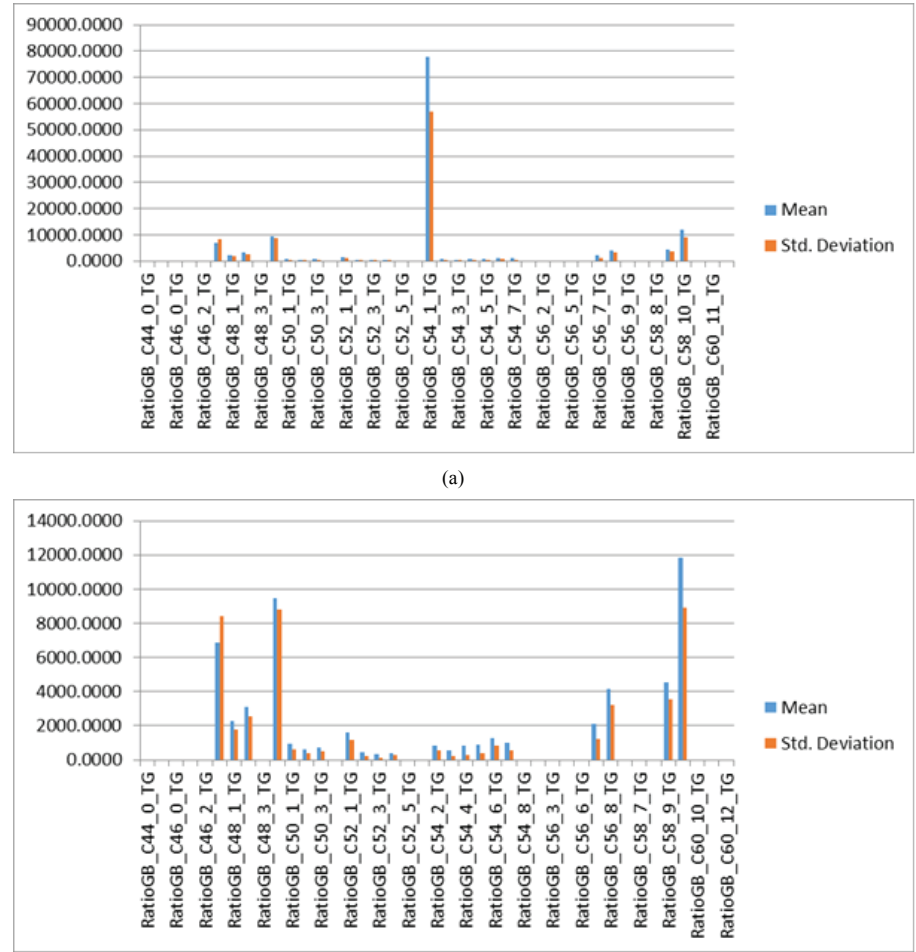

Figure 3: With depicted ration [brain-TGs]/[blood-TGs] for grey neocortical matter. When from Figure 3a (top) the dominant TG-C:54-1 peak $\approx 78000.0$ is eliminated we get in Figure $3 \mathrm{~b}$ (bottom) variety of TGs fatty acids fatty acids of varying degrees of unsaturation and chain length which possibly fuel the brain. Mean (blue) and red (std) of 8 control human subjects.

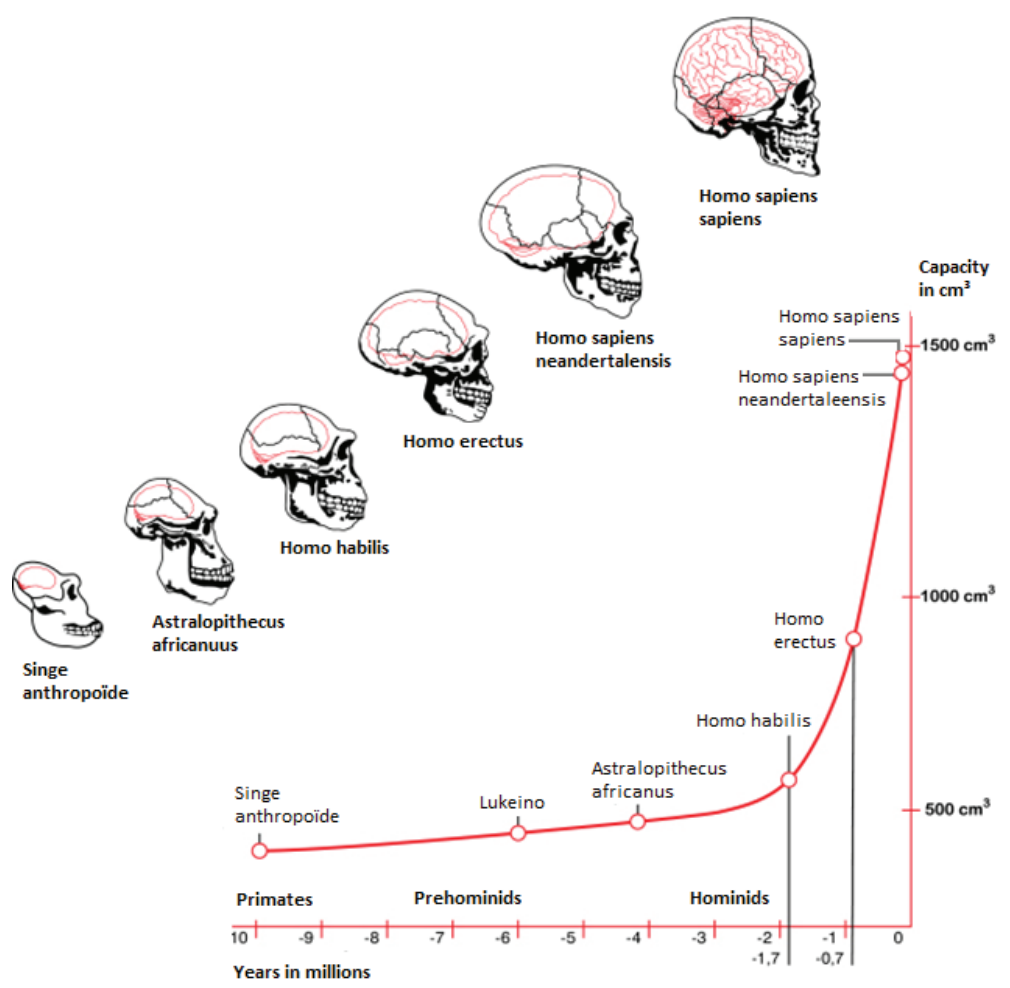

Figure 4: Human evolution based on skull endocasts of fossil archaic primates and early hominids. We hypothesize Triacylglycerols (TGs) were the trigger for human encephalization which was the growth factor for logarithmic skull expansion from Homo erectus towards Homo sapiens during course of evolution (Figure 1) [11] 
Citation: Ginneken VV, Meerveld AV, Verheij E, Greef JVD (2017) On the Futile Existence of DHA, None of EPA and the Predominant Role of the Triacylglycerols (TGs) in the Post Mortem Human Brain: An LCMS Study with Evolutionary Implications. J Bioanal Biomed 9: 152-158. doi:10.4172/1948-593X.1000170

\begin{tabular}{|c|c|c|c|c|c|}
\hline Compound & Control-white brain Mean \pm SD $(n=8)$ & $\%$ & Control-grey brain Mean \pm SD $(n=8)$ & $\%$ & T-test P-value \\
\hline$\sum$-LPC & $0.412 \pm 0.1022$ & $0.30 \%$ & $0.250 \pm 0.0498$ & $0.20 \%$ & $\mathrm{P} \leq 0.0024^{* *}$ \\
\hline$\sum$-SPM & $8.731 \pm 1.190$ & $6.32 \%$ & $3.039 \pm 0.6128$ & $2.46 \%$ & $P \leq 0.00000001^{\star \star \star *}$ \\
\hline$\sum$-DG & $0.457 \pm 0.1472$ & $0.33 \%$ & $0.074 \pm 0.0237$ & $0.06 \%$ & $P \leq 0.0001^{* \star *}$ \\
\hline$\sum-\mathrm{PC}$ & $25.773 \pm 1.355$ & $18.65 \%$ & $23.385 \pm 2.1260$ & $18.90 \%$ & $P \leq 0.0202^{*}$ \\
\hline$\sum-\mathrm{PE}$ & $1.680 \pm 0.1719$ & $1.22 \%$ & $1.692 \pm 0.3036$ & $1.38 \%$ & $P \leq 0.926$ \\
\hline$\sum$-PC-plasmalogens & $2.651 \pm 0.1833$ & $1.92 \%$ & $0.869 \pm 0.5206$ & $0.70 \%$ & $\mathrm{P} \leq 0.00000001^{* * * *}$ \\
\hline$\sum$-PE-plasmalogens & 3.9360 .4561 & $2.85 \%$ & $1.034 \pm 0.5453$ & $0.84 \%$ & $P \leq 0.000000021^{* * * *}$ \\
\hline$\sum$-ChE & $0.069 \pm 0.0053$ & $0.05 \%$ & $0.063 \pm 0.0053$ & $0.05 \%$ & $P \leq 0.0463$ \\
\hline$\sum$-TG & $94.419 \pm 26.4829$ & $68.32 \%$ & $93.971 \pm 24.7624$ & $75.96 \%$ & $P \leq 0.9682$ \\
\hline$\sum$-Total & $138.200 \pm 26.765$ & $\approx 100 \%$ & $123.712 \pm 23.6108$ & $\approx 100 \%$ & $P \leq 0.2704$ \\
\hline DGLA (C20:3n6) & $0.0022 \pm 0.0004$ & $0.00159 \%$ & $0.0014 \pm 0.0003$ & $0.00113 \%$ & $P \leq 0.0007^{* *}$ \\
\hline AA (C:20-4-ChE) & $0.008 \pm 0.0015$ & $0.00579 \%$ & $0.007 \pm 0.0016$ & $0.00565 \%$ & $P \leq 0.0422^{*}$ \\
\hline EPA (C:20-5-ChE) & Not detected & - & Not detected & - & - \\
\hline DHA (C:22-6-ChE) & $0.0002 \pm 0.0003$ & $0.00014 \%$ & $0.002 \pm 0.0005$ & $0.00162 \%$ & $P \leq 0.2129$ \\
\hline AA/DHA & $5.16 \pm 1.051$ & - & $3.44 \pm 0.638$ & - & $P \leq 0.0021^{* *}$ \\
\hline AA/EPA & - & - & - & - & - \\
\hline Compound & $\begin{array}{l}\text { Diabetes2-white brain } \\
\text { Mean } \pm S D(n=8)\end{array}$ & & $\begin{array}{l}\text { Diabetes-2-grey brain } \\
\text { Mean } \pm \operatorname{SD}(n=8)\end{array}$ & & P-value \\
\hline$\sum$-LPC & $0.338 \pm 0.0868$ & $0.22 \%$ & $0.222 \pm 0.0434$ & $0.14 \%$ & $P \leq 0.0069^{* *}$ \\
\hline$\sum$-SPM & $8.978 \pm 2.5746$ & $5.81 \%$ & $3.246 \pm 0.4956$ & $2.07 \%$ & $P \leq 0.000253^{* * *}$ \\
\hline$\sum$-DG & $0.440 \pm 0.21419$ & $0.29 \%$ & $0.078 \pm 0.0376$ & $0.05 \%$ & $P \leq 0.0018^{\star *}$ \\
\hline$\sum-\mathrm{PC}$ & $24.568 \pm 0.906$ & $15.91 \%$ & $23.393 \pm 0.971$ & $14.94 \%$ & $P \leq 0.025346^{*}$ \\
\hline$\sum-\mathrm{PE}$ & $1.692 \pm 0.3036$ & $1.09 \%$ & $1.018 \pm 0.0839$ & $0.66 \%$ & $P \leq 0.0003^{* * *}$ \\
\hline$\sum$-PC-plasmalogens & $2.599 \pm 0.5507$ & $1.68 \%$ & $0.805 \pm 0.5707$ & $0.51 \%$ & $P \leq 0.0000001^{* * * *}$ \\
\hline$\sum$-PE-plasmalogens & $3.960 \pm 1.0,552$ & $2.56 \%$ & $0.9814 \pm 0.57105$ & $0.63 \%$ & $P \leq 0.0000001^{* \star * *}$ \\
\hline$\sum-\mathrm{ChE}$ & $0.0780 \pm 0.0130$ & $0.05 \%$ & $0.069 \pm 0.0097$ & $0.44 \%$ & $P \leq 0.1172$ \\
\hline$\sum$-TG & $112.592 \pm 28.9164$ & $72.90 \%$ & $126.715 \pm 54.6613$ & $80.93 \%$ & $P \leq 0.5320$ \\
\hline$\sum$-Total & $154.439 \pm 28.0851$ & $\approx 100 \%$ & $156.576 \pm 52.7645$ & $\approx 100 \%$ & $P \leq 0.9253$ \\
\hline DGLA (C20:3n6) & $0.00258 \pm 0.00079$ & $0.00167 \%$ & $0.00128 \pm 0.00032$ & $0.00082 \%$ & $P \leq 0.0018^{* *}$ \\
\hline AA (C:20-4-ChE) & $0.01041 \pm 0.00396$ & $0.0067 \%$ & $0.00765 \pm 0.00252$ & $0.0049 \%$ & $P \leq 0.1227$ \\
\hline EPA (C:20-5-ChE) & Not detected & - & Not detected & - & - \\
\hline DHA (C:22-6-ChE) & $0.00172 \pm 0.00014$ & $0.0011 \%$ & $0.00206 \pm 0.00024$ & $0.0013 \%$ & $P \leq 0.005^{\star *}$ \\
\hline AA/DHA & $6.14 \pm 2.41$ & - & $3.68 \pm 0.927$ & - & $P \leq 0.0244^{* *}$ \\
\hline AA/EPA & - & - & - & - & - \\
\hline
\end{tabular}

Table 2: Summarized relative concentrations determined with LC-MS techniques of the different lipid compounds in human brain homogenate (white vs. grey) of two patients groups: one control-group ( $\mathrm{N}=8)$ and one Diabetes-2 group ( $\mathrm{N}=8)$. DGLA: Dihomo-y-linolenic Acid; AA: Arachidonic Acid; EPA: Eicosapentaenoic Acid; DHA: Docosahexaenoic Acid.

states and this might be stimulated by a physiological level of Arachidonic Acid (ARA) [13]. But probably many other compounds of mainly the Cholesteryl fraction (PUFAs) are involved, and for this reasoning we will elucidate this topic related to optional passive/active carriers in the BBB. Recently, the opinion is contradicted that Very Long Chain Fatty Acids (VLCFA) can pass the BBB to fuel the brain [14]. Our findings are opposite and give awareness of a predominant role of the TGs.

From our observations the TG C:54-1 (Figure 3a) can be considered-following our "membrane-model"- the most "leaky" position in the BBB which can be based on several TGs combinations like: a) Two times C18:0 stearate; b) One times C18:1 oleate (SSO); or c) The combination C16:0; C18:0 and C20:0. If we eliminate this peak a whole range smaller C:46-C:60 TGs lipid molecules are possibly fueling the brain according to our observations. These are in contrast towards the theories of considering the ability of VLCFA to fuel the brain [14]. Following our "membrane" approach the transporter for "fishy" PUFA, DHA is not detected which is in contrast to the research of the studies of Nguyen et al.; Ben-Zvi et al.; Guemez-Gamboa et al. [4,16,17], focusses on the Mfsd2a protein as "leaky position" in the BBB for the "fishy" omega-3 DHA.

In addition, the perception consists that some systemic fatty acids are likely to cross the human Blood-Brain Barrier (BBB) and thereby influence central fatty acid concentrations [18]. However, our data are indicative that the TGs are the major constituents passing the $\mathrm{BBB}$ and consequently we hypothesize that the quantitative aspect of encephalization has to come from the TGs (Table 2).

The biological activities of omega-3 fatty acids (n-3 FAs) have been under extensive study for several decades and its role in brain functioning and mental health is beyond scope of extremely importance from qualitative perception $[19,20]$. An abundance of the Polyunsaturated Fatty Acid (PUFAs)-among them DHA in seafoodhas been suggested as being helpful in the development of a large brain [20]. The harvesting, processing, and consuming of seafood are ancient practices with archaeological evidence dating back well into the Paleolithic [21]. In contrast, other researchers claim a terrestrial diet could also have provided the necessary DHA [21]. The latter vision we support, considering the futile existence of DHA and complete lack of 
Citation: Ginneken VV, Meerveld AV, Verheij E, Greef JVD (2017) On the Futile Existence of DHA, None of EPA and the Predominant Role of the Triacylglycerols (TGs) in the Post Mortem Human Brain: An LCMS Study with Evolutionary Implications. J Bioanal Biomed 9: 152-158. doi:10.4172/1948-593X.1000170

EPA in human brain.. Presently, there is an important dichotomous approach in evolutionary sciences which lipid fraction plays a major role in human encephalization: the omega-3 PUFAs or the TGs. Earlier we described the observation in a C56bl6 High-Fat diet induced obese mouse model on bovine lard with large amounts of unsaturated TGs C50:1; C:50-2; C:52-2; C:54-3; C:54-4 and C:56-3 TGs might have played a role in mammalian encephalization [5,11]. Our observations support this new insight that TGs $(\approx \mathrm{VLCFA})$ can pass the BBB and fuel the modern human brain. On the other hand during course of human evolution these TGs were the trigger for human encephalization which was the growth factor for logarithmic skull expansion from Homo erectus towards Homo sapiens during course of evolution [11] ] (Figure 1 and 4).

With the expected pandemic of about one billion people with a form of dementia or mild-Alzheimer around 2030 [22], our findings can also be applied in finding appropriate nutrition and pharma with more emphasis on TGs to respond to this wave of mental disorders among our elderly.

\section{References}

1. Ramachandran VS (2012) The tell-tale brain; unlocking the mystery of human nature. Windmill Books, London, p: 357.

2. Kaas JH (2012) The evolution of neocortex in primates. Prog Brain Res 195 91-102.

3. van der Greef J (2011) Perspective: all systems go. Nature 480: s87.

4. Nguyen LN, Ma D, Shui G, Wong P, Cazenave-Gassiot A, et al. (2014) Mfsd2a is a transporter for the essential omega-3 fatty acid docosahexaenoic acid. Nature 509: 503-506.

5. van Ginneken V, de Vries E, Verheij E, van der Greef J (2017) "Brain steatosis" in an obese mouse model during cycles of Famine and Feast: the underestimated role of fat (WAT) in brain volume formation. Integr Mol Med 4.

6. van Ginneken V, Meerveld AV, Wijgerde T, van der Greef J (2017) Hypothesis: Hunter-prey correlation between migration routes of African buffaloes and early hominids: Evidence for the "Out of Africa" hypothesis. Integr Mol Med 4: 1-5.

7. Chang CY, Ke DS, Chen JY (2009) Essential fatty acids and human brain. Acta Neurol Taiwan 18: 231-241.

8. Carrié I, Clément $M$, de Javel D, Francès H, Bourre JM (2000) Specific phospholipid fatty acid composition of brain regions in mice: effects of $n-3$ polyunsaturated fatty acid deficiency and phospholipid supplementation. J Lipid Res 41: 465-472.
9. Chrast R, Saher G, Nave KA, Verheijen MH (2011) Lipid metabolism in myelinating glial cells: lessons from human inherited disorders and mouse models. J Lipid Res 52: 419-434.

10. O'Brien JS, Sampson EL (1965) Lipid composition of the normal human brain grey matter, white matter, and myelin. J Lipid Res 6: 537-544.

11. Stringer CB (1994) Evolution of early humans. In: Jones S, Martin R, Pilbeam D (eds.) The Cambridge Encyclopedia of Human Evolution. Cambridge: Cambridge University Press, p: 242.

12. van Ginneken V, Verheij E, Hekman M, van der Greef J (2017) Characterization of the lipid profile post mortem for Type-2 diabetes in human brain and plasma of the elderly with LCMS-techniques: a descriptive approach of diabetic encephalopathy. Integr Mol Med 4: 1-10.

13. Murphy EJ (2015) Blood-brain barrier and brain fatty acid uptake: Role of arachidonic acid and PGE2. J Neurochem 135: 845-848.

14. Schönfeld P, Reiser G (2013) Why does brain metabolism not favor burning of fatty acids to provide energy? Reflections on disadvantages of the use of free fatty acids as fuel for brain. J Cereb Blood Flow Metab 33: 1493-1499.

15. Lagarde M, Bernoud N, Brossard N, Lemaitre-Delaunay D, Thiès F, et al. (2001) Lysophosphatidylcholine as a preferred carrier form of docosahexaenoic acid to the brain. J Mol Neurosci 16: 215-221.

16. Ben-Zvi A, Lacoste B, Kur E, Andreone BJ, Mayshar Y, et al. (2014) Mfsd2a is critical for formation and function of the blood-brain barrier. Nature 509: 507-511.

17. Guemez-Gamboa A, Nguyen LN, Yang H, Zaki MS, Kara M, et al. (2015) Inactivating mutations in MFSD2A, required for omega-3 fatty acid transport in brain, cause a lethal microcephaly syndrome. Nat Genet 47: 809-813.

18. Guest J, Garg M, Bilgin A, Grant R (2013) Relationship between central and peripheral fatty acids in humans. Lipids Health Dis 12: 79.

19. Crawford MA, Bloom M, Broadhurst CL, Schmidt WF, Cunnane SC, et al. (2000) Evidence for the unique function of docosahexaenoic acid (DHA) during the evolution of the modern hominid brain. Lipids 34: S39-S47.

20. Broadhurst CL, Wang Y, Crawford MA, Cunnane SC, Parkington JE, et al. (2002) Brain-specific lipids from marine lacustrine, or terrestrial food resources: potential impact on early African Homo sapiens. Comp Biochem Physiol B Biochem Mol Biol 131: 653-673.

21. Carlson BA, Kingston JD (2007) Docosahexaenoic acid biosynthesis and dietary contingency: Encephalization without aquatic constraint. Am J Hum Bio 19: $585-588$

22. Alzheimer Association Report (2013) Alzheimer's disease facts and figures. Alzheimers Dement 9: 208-245. 